\title{
Coin-Based Anonymous Fingerprinting
}

\author{
Birgit Pfitzmann and Ahmad-Reza Sadeghi \\ Universität des Saarlandes, Fachbereich Informatik, \\ D-66123 Saarbrücken, Germany \\ \{pfitzmann, sadeghi\}@cs.uni-sb.de
}

\begin{abstract}
Fingerprinting schemes are technical means to discourage people from illegally redistributing the digital data they have legally purchased. These schemes enable the original merchant to identify the original buyer of the digital data. In so-called asymmetric fingerprinting schemes the fingerprinted data item is only known to the buyer after a sale and if the merchant finds an illegally redistributed copy, he obtains a proof convincing a third party whom this copy belonged to. All these fingerprinting schemes require the buyers to identify themselves just for the purpose of fingerprinting and thus offer the buyers no privacy. Hence anonymous asymmetric fingerprinting schemes were introduced, which preserve the anonymity of the buyers as long as they do not redistribute the data item.

In this paper a new anonymous fingerprinting scheme based on the principles of digital coins is introduced. The construction replaces the general zero-knowledge techniques from the known certificate-based construction by explicit protocols, thus bringing anonymous fingerprinting far nearer to practicality.
\end{abstract}

\section{Introduction}

Fingerprinting schemes are cryptographic techniques supporting the copyright protection of digital data. They do not require tamper-resistant hardware, i.e., they do not belong to the class of copyright protection methods which prevent copying. It is rather assumed that buyers obtain data in digital form and can copy them. Buyers who redistribute copies disregarding the copyright conditions are called traitors. Fingerprinting schemes discourage traitors by enabling the original merchant to identify a traitor who originally purchased the data item. Every sold copy is slightly different from the original data item and unique to its buyer. Obviously the differences to the original represent the information embedded in the data item, which must be imperceptible. As several traitors might collude and compare their copies to find and eliminate the differences, cryptographic methods were used to make fingerprinting schemes collusion tolerant. There are different classes of fingerprinting schemes called symmetric [BMP86, BS95] and asymmetric [PS96, PW97a, BM97]. In contrast to symmetric schemes, asymmetric schemes require the data item to be fingerprinted via an interactive protocol between the buyer and the merchant where the buyer also inputs her own secret. At the end of this protocol only the buyer knows the fingerprinted data 
item. However, after finding a redistributed copy the merchant can extract information which not only enables him to identify a traitor but also provides him with a proof of treachery that convinces any third party. The main construction in PS96] was based on general primitives; an explicit construction, i.e., without such primitives, was only given for the case without significant collusions. Explicit collusion-tolerant constructions were given in [PW97a, BM97. A special variant of fingerprinting is traitor tracing [CFN94, NP98; here the keys for broadcast encryption [FN94 are fingerprinted. Asymmetric traitor tracing was introduced in [Pfi96] with a construction based on general primitives. Explicit constructions for this case were also given in PW97a]. Even more efficient construction were given in KD98; however, they are not asymmetric in the usual sense but "arbitrated", i.e., a certain number of predefined arbiters can be convinced by the merchant (similar to the difference between arbitrated authentication codes and asymmetric signature schemes).

As in "real-life" market places, it is desired that electronic market places offer privacy to the customers. It should be possible to buy different articles (pictures, books, etc.) anonymously, since buying items can reveal a lot of behavioristic information about an individual. To allow this also for buying fingerprinted items, anonymous asymmetric fingerprinting schemes were proposed [PW97b]. Note that in normal fingerprinting (symmetric and asymmetric) the buyer has to identify herself during each purchase. In anonymous fingerprinting the anonymity of the buyers is preserved as long as they do not redistribute copies of the data item.

In this paper, we introduce a new anonymous asymmetric fingerprinting scheme based on the principles of digital coins. Our protocols are explicit, in contrast to the scheme in [PW97b], where general theorems like "every NPlanguage has a zero-knowledge proof system" were used, and thus far more efficient. The anonymity is information-theoretic and security computational. Security of one party relies on a restrictiveness assumption about the underlying payment system, which we formulate precisely.

\section{The Model of Anonymous Fingerprinting}

The involved parties in the model are merchants $\mathcal{M}$, buyers $\mathcal{B}$, registration centers $\mathcal{R C}$ and arbiters $\mathcal{A}$. For the purpose of fingerprinting it is required in this model that buyers register themselves to a registration center $\mathcal{R C}$ (e.g., their bank). The required trust in $\mathcal{R C}$ should be minimum such that a cheating $\mathcal{R C}$ can only refuse a registration 1 It is assumed that $\mathcal{B}$ can generate signatures (using an arbitrary signature scheme) under her "real" identity $I D_{\mathcal{B}}$ and that the corresponding public keys have already been distributed. Furthermore there

\footnotetext{
${ }^{1}$ In particular, even a collusion of $\mathcal{M}$ and $\mathcal{R C}$ should not be able to trace a buyer who did not redistribute a data item. Otherwise one can trivially use any known nonanonymous asymmetric scheme and simply let the initial key pair of the buyer be certified under a pseudonym whose owner is only known to the certification authority. This was overlooked in [Dom98].
} 
is no special restriction on the arbiter $\mathcal{A}$. Any third party having access to the corresponding public keys should be convinced by the proof. The main subprotocols of the construction are registration, fingerprinting, identification, and trial. Identification includes a variant "enforced identification" for the case where $\mathcal{R C}$ refuses to cooperate. The main security properties are:

- Security for the merchant: As long as collusions do not exceed a certain size, the merchant will be able to identify a traitor for each illegally redistributed item and to convince any honest arbiter. (In case $\mathcal{R C}$ colludes with the traitors the identified traitor may be $\mathcal{R C}$.)

- Security for the buyer and $\mathcal{R C}$ : Nobody is unduly identified as a traitor; at least no honest arbiter will believe it.

- Anonymity as sketched above; different purchases by one buyer should also be unlinkable.

For the detailed definitions of the subprotocols and security properties we refer the interested reader to $\mathrm{PW97b}$.

\section{Overview of the Construction}

To see more precisely what we achieve, note that PW97b contains a modular construction: The first part, called framework, is a construction based on certificates. At the end, the merchant holds a commitment com to a certain value $\mathrm{emb}$ and possibly other information. The buyer can open the commitment and may also hold other information. This framework guarantees that whenever the merchant later obtains $e m b$, he can identify this buyer and win a trial against him. In the second part, the value $e m b$ is embedded into the data item in a way that does not release additional information about $\mathrm{emb}$. The embedding procedure must guarantee that whenever a collusion of at most the maximum tolerated size redistributes a data item, the merchant will be able to reconstruct the value $e m b$ that was used by at least one traitor. For the second part, constructions were previously only known for the case of traitor tracing and for normal fingerprinting without collusion tolerance. The latter is explicit, and one can see quite easily that the former (based on PW97a Section 4) can also be made explicit by using the efficient key selection protocol from Section 2.3 of the same paper in the appropriate places. The main technical part in [PW97b] was to construct a suitable collusion-tolerant embedding procedure for normal fingerprinting.

In contrast, the framework part in PW97b is a comparatively simple construction where $e m b$, the content of the commitment, is a signature with respect to a key that the merchant must not know, and the buyer proves with a general zero-knowledge technique that she knows such a key and a certificate by $\mathcal{R C}$ on it. It is this first part that we replace with the explicit and much more efficient coinbased construction. It can then be combined with the known second parts. This gives us explicit overall constructions for collusion-tolerant anonymous traitor tracing and for anonymous normal fingerprinting without collusion tolerance. For collusion-tolerant normal fingerprinting, the construction in [PW97b] is not 
explicit, but it is mentioned that all the steps in a secure 2-party computation look quite simple so that it should be possible to find simpler explicit realizations for them. This can in fact be done (each time exploiting either homomorphism - note that even the Reed-Solomon codes are a linear operation - or the efficient table-lookup from [PW97b], end of Section 2.3), but we do not attempt to include details of that here.

The basic idea for using digital cash systems with double-spender identification to construct an anonymous fingerprinting scheme is as follows: Registration will correspond to withdrawing a coin. (The "coins" serve only as a cryptographic primitive and have no monetary value.) The untraceability of the cash system will give us the unlinkability of the views of the registration center and the merchant. Redistribution of a data item should correspond to double-spending of the underlying coin, i.e., the value $e m b$ embedded in the data item will be similar to the response in a second payment with the coin. We could execute a complete first payment during fingerprinting, but actually our protocols can be simpler. (They are more like "zero-spendable" coins where each coin as such can be shown, but any response to a challenge leads to identification.) One new problem is that while in a payment the response is given to the merchant in clear, in our case it must be verifiably hidden in a commitment. Another problem is that double-spender identification is usually a binary decision. In our case, however, it must be decided reliably under what copyright conditions the redistributed item was bought - e.g., there may be items that can be redistributed after a while. In the formal security requirements this is a value text input in fingerprinting and also in a trial. Thus the identification information must be linked to such a text during fingerprinting in a way that even a collusion of a merchant and the registration center cannot forge, although such a collusion can sign additional coins that look like belonging to a specific buyer.

\section{Construction}

Our explicit construction employs the ideas from the digital cash scheme in [Bra94], or, as we do not have the same double-spender identification, at least from the underlying blind signature scheme [CP93. We make the following conventions:

Algebraic Structure: All arithmetic operations are performed in a group $G_{q}$ of order $q$ for which efficient algorithms are known to multiply, invert, determine equality of elements, test membership and randomly select elements. Any group $G_{q}$ satisfying these requirements and in which the computation of discrete logarithms is infeasible can be a candidate. For concrete constructions one can assume that $G_{q}$ is the unique subgroup of prime order $q$ of the multiplicative group $\mathbb{Z}_{p}^{*}$ where $p$ is a prime such that $q \mid(p-1)$.

Hash Function: Hash functions are denoted by hash. We have to make the same assumptions as in Bra94, which is behavior similar to a random oracle. 
Commitment Scheme: We use two commitment schemes. The first one is based on discrete logarithms in $G_{q}$ (see BKK90 for the one-bit version and CHP92, Ped92 based on BCP88 for the general version). To commit to a value $b \in \mathbb{Z}_{q}$, the committer needs generators $g^{\prime \prime}, h^{\prime \prime} \in_{R} G_{q} \backslash\{1\}$, which are typically randomly generated and sent by the recipient. Then the committer selects $x \in_{R}$ $\mathbb{Z}_{q}$ and computes the commitment $y=B C^{D L}(x, b)=g^{\prime \prime b} h^{\prime \prime x} \bmod p$. To open $y$, the committer reveals $(b, x)$. This scheme is information-theoretically hiding and it is binding under the discrete logarithm assumption in the corresponding group. Moreover due to the homomorphic property of this scheme one can commit to a number $r \in \mathbb{Z}_{q}^{*}$ using commitments to the bits of $r$ : Let $r=\sum_{j=0}^{l-1} r_{j} 2^{j}$ be the binary representation of $r$ and $B C^{D L}\left(x_{j}, r_{j}\right)$ be commitments to the bits. Then $\prod_{j=0}^{l-1}\left(B C^{D L}\left(x_{j}, r_{j}\right)\right)^{2^{j}}=g^{\prime \prime r} h^{\prime \prime x}=B C^{D L}(x, r) \bmod p$ with $x=$ $\sum_{j=0}^{l-1} x_{j} 2^{j} \bmod q$.

The second bit commitment scheme is based on quadratic residues (see GM84, BCC88). To commit to a bit $b^{\prime}$ the committer computes $y=$ $B C^{Q R}\left(x^{\prime}, b^{\prime}\right)=(-1)^{b^{\prime}}{x^{\prime}}^{2} \bmod n$ where $x^{\prime} \in \in_{R} \mathbb{Z}_{n}^{*}$ and $n$ is a Blum integer chosen by the committer.

\subsection{Key Distribution and Registration}

Registration Center Key Distribution: $\mathcal{R C}$ randomly selects a group $G_{q}$ and generators $g, g_{1}, g_{2} \in_{R} G_{q} \backslash\{1\}$ and a number $x \in_{R} \mathbb{Z}_{q}^{*}$ as its secret key. $\mathcal{R C}$ also chooses a hash function hash. It publishes the group description, $\left(g, g_{1}, g_{2}\right)$, its public key $h \equiv g^{x} \bmod p$ and hash.

The correctness of the group description (i.e., that $p$ and $q$ primes with $q \mid(p-1)$ and generated in a way believed to exclude trap doors) and whether the generators are elements of $G_{q} \backslash\{1\}$ should be verified by other parties when using them. However, other parties do not rely on the randomness of the generators.

Opening a one-time account: This phase is similar to [Bra94, but in order to bind the identification information to a specific purchase text, each "account" is used only once. To open an account, $\mathcal{B}$ chooses $i \in \in_{R} \mathbb{Z}_{q}^{*}$ randomly and secretly and computes $h_{1} \equiv g^{i} \bmod p\left(\right.$ with $\left.h_{1} g_{2} \neq 1\right)$. She gives $h_{1}$ to $\mathcal{R C}$ and proves that she knows $i$ (using the zero-knowledge proof from [CEG88 or, more efficiently, Schnorr identification Sch91]). First $\mathcal{R C}$ verifies that the account number $h_{1}$ has not been used before. Then it stores $h_{1}$ in its registration database together with the claimed normal identity $I D_{\mathcal{B}}$ of this buyer. $\mathcal{B}$ gives a signature $\operatorname{sig} g_{\text {coin }}$ on $h_{1}$ (with a suitable explanation) under her normal identity $I D_{\mathcal{B}}$ and $\mathcal{R C}$ verifies it. This signature can be used in later trials to show that $\mathcal{B}$ is responsible for this "account number" $h_{1}$.

Withdrawal: The protocol is shown in Figure 1 Essentially, $\mathcal{R C}$ signs the common input $m \equiv h_{1} g_{2} \equiv g_{1}^{i} g_{2} \bmod p$ using a restrictive blind signature as in [Bra94. Thus $\mathcal{B}$ obtains a signature $\sigma^{\prime}=\left(z^{\prime}, a^{\prime}, b^{\prime}, r^{\prime}\right)$ on $m^{\prime} \equiv m^{s} \equiv g_{1}^{i s} g_{2}^{s} \bmod p$ where $s \in \in_{R} \mathbb{Z}_{q}^{*}$ is chosen randomly and secretly by $\mathcal{B}$. 
Our protocol is also similar to the withdrawal protocol in Bra94 in that an additional value is included in the hashing to obtain the challenge $c^{\prime}$ and thus in the signing process. In our case it is the public key of the key pair $\left(s k_{t e x t}, p k_{\text {text }}\right)$ from an arbitrary signature scheme, here Schnorr's for concreteness [Sch9]. We call the triple $\left(m^{\prime}, p k_{t e x t}, \sigma^{\prime}\right)$ a coin.

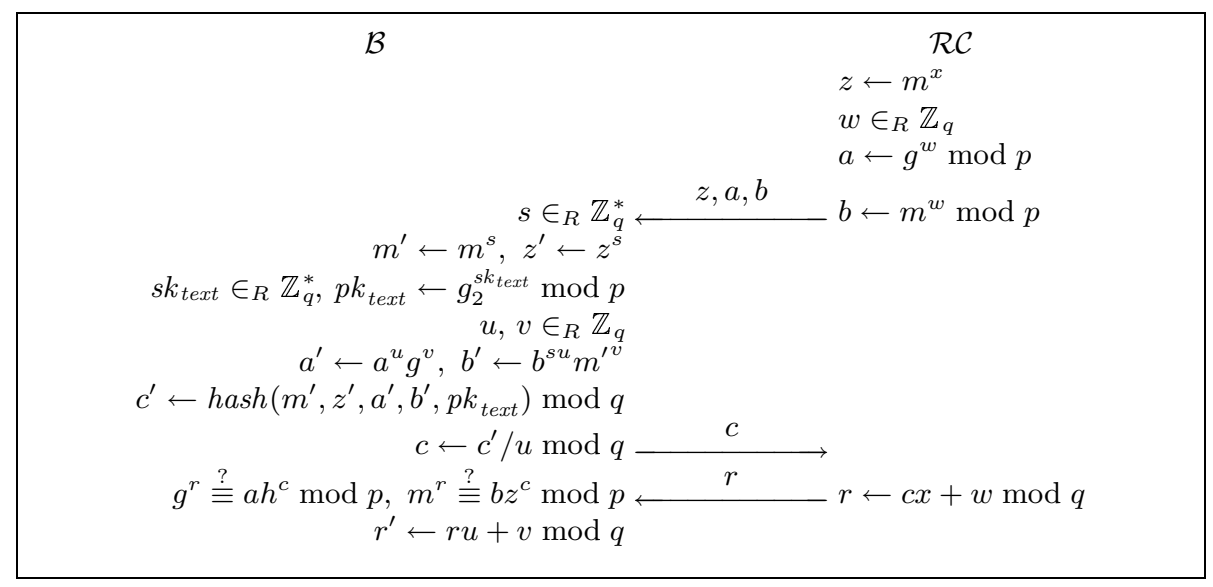

Fig. 1. The Withdrawal Part of the Registration Protocol

\subsection{Fingerprinting}

The fingerprinting subprotocol is executed between the (anonymous) buyer $\mathcal{B}$ and the merchant $\mathcal{M}$. This protocol differs (except for Step 1) from the payment protocol in Bra94. The common input is a text, text, describing the purchase item and licensing conditions.

Step 1: $\mathcal{B}$ selects an unused coin $\operatorname{coin}^{\prime}=\left(m^{\prime}, p k_{\text {text }}, \sigma^{\prime}\right)$. She uses the corresponding $s k_{\text {text }}$ to make a Schnorr signature $\operatorname{sig}_{\text {text }}$ on text and sends $\left(\operatorname{coin}^{\prime}\right.$, sig $\left._{\text {text }}\right)$ to $\mathcal{M}$. Now $\mathcal{M}$ first verifies the validity of $\operatorname{coin}{ }^{\prime}$ by computing $c^{\prime} \equiv \operatorname{hash}\left(m^{\prime}, z^{\prime}, a^{\prime}\right.$, $\left.b^{\prime}, p k_{\text {text }}\right)$ mod $q$ and checking whether $m^{\prime} \neq 1$ and $g^{r^{\prime}} \equiv a^{\prime} h^{c^{\prime}}$ and $m^{r^{\prime}} \equiv b^{\prime} z^{\prime c^{\prime}}$ $\bmod p$ hold Bra94. We say that a coin is valid if and only if it passes these tests. He then verifies $s i g_{\text {text }}$ using $p k_{\text {text }}$ from coin'.

Step 2: $\mathcal{B}$ takes the internal structure $(i s, s)$ of $m^{\prime} \equiv g_{1}^{i s} g_{2}^{s}$ as the value to be embedded in the data item. Hence $e m b=(i s, s)$, and let $r_{1}-i s, r_{2}=s$.

Since $\mathcal{M}$ should not get any useful information on this value, $\mathcal{B}$ hides it in a commitment. While the certificate-based framework in [PW97b could leave the type of commitment open, we have to provide an efficient link from the given representation of $e m b$, i.e., $\mathrm{m}^{\prime}$, to the type of commitments needed in the later 
embedding procedures. For normal fingerprinting with and without collusion tolerance, these are quadratic residue commitments to individual bits of emb?

We start this by producing discrete logarithm commitments to a binary representation of $r_{1}$ and $r_{2}$ : The merchant $\mathcal{M}$ sends generators $g^{\prime \prime}$ and $h^{\prime \prime}$ to $\mathcal{B}$, and $\mathcal{B}$ sends back commitments $\operatorname{com}_{k j}=g^{\prime \prime r_{k j}} h^{\prime \prime x_{k j}}$ where $r_{k}=\sum_{j=0}^{l-1} r_{k j} 2^{j}$ for $k=1,2$ and $x_{k j} \in_{R} \mathbb{Z}_{q}$. $\mathcal{M}$ may choose the generators randomly once for all its buyers. $\mathcal{B}$ should verify that they are elements of $G_{q} \backslash\{1\}$. From these individual commitments, $\mathcal{M}$ computes the commitments to $r_{1}$ and $r_{2}$, i.e., $\operatorname{com}_{k}=\prod_{j=0}^{l-1}\left(\operatorname{com}_{k j}\right)^{2^{j}}$ for $k=1,2$. Now $\mathcal{B}$ proves the following predicate $P$ to $\mathcal{M}$ : The content of the commitments $c o m_{k}$ is a pair $\left(r_{1}, r_{2}\right) \in \mathbb{Z}_{q}^{*} \times \mathbb{Z}_{q}^{*}$ with $m^{\prime} \equiv g_{1}^{r_{1}} g_{2}^{r_{2}} \bmod p$. This can be done in zero-knowledge as shown in Figure 图, similar to other proofs concerning knowledge of representations of numbers with respect to certain generators following [EG88]. As usual, we could also use larger challenges $c$ at the cost of the real zero-knowledge property.

Note that this protocol does not prove that the values $r_{k j}$ are binary; such a proof will be a side effect of Step 3 .

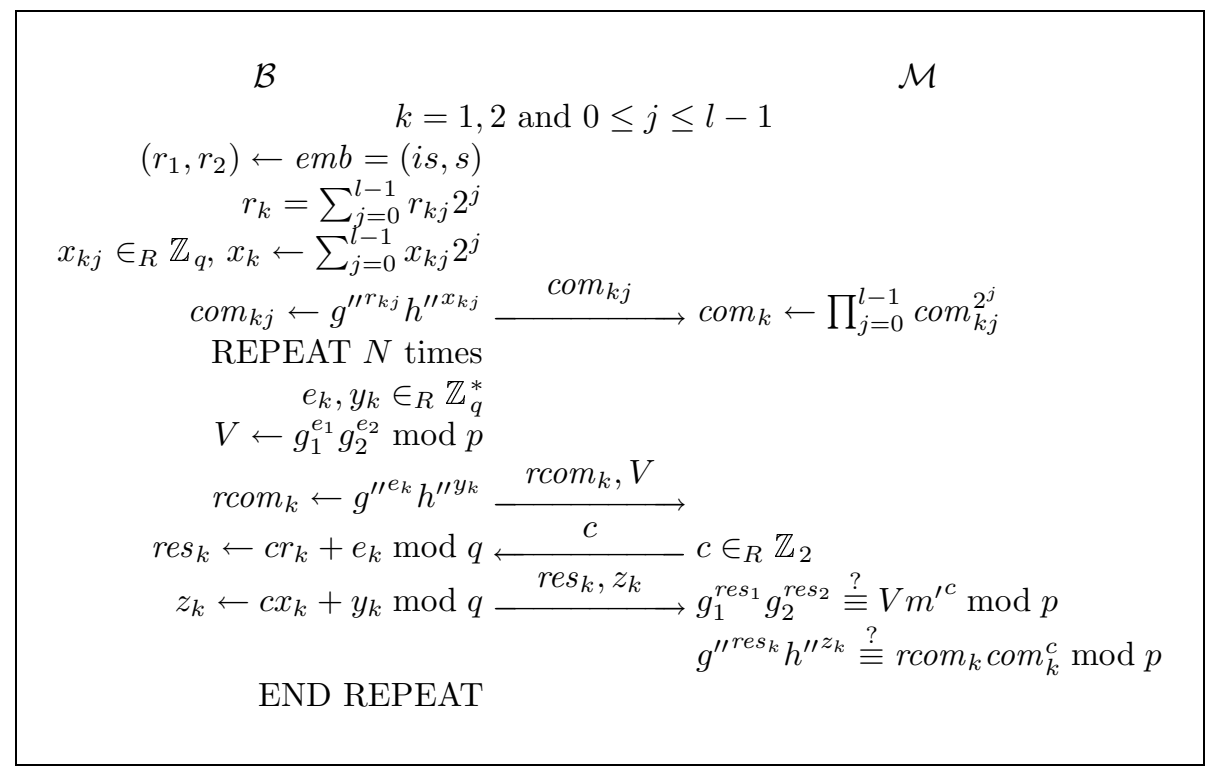

Fig. 2. Step 2 of the Fingerprinting Protocol

Step 3: Now $\mathcal{B}$ additionally computes quadratic residue commitments on the same values $r_{k j}$. As mentioned, these are needed as input to the embedding

${ }^{2}$ For traitor tracing, they are quadratic residue commitments to small blocks of emb represented in unary. Such a representation can also be derived efficiently from commitments to the bits. 
procedures. We denote them by $\operatorname{com}_{k j}^{\prime}=B C^{Q R}\left(x_{k j}^{\prime}, r_{k j}\right)$ for $x_{k j}^{\prime} \in_{R} \mathbb{Z}_{n}^{*}$, where $n$ is a Blum integer chosen by the buyer. $\mathcal{B}$ sends these commitments to $\mathcal{M}$ and proves in zero-knowledge that the contents in each pair $\left(c o m_{k j}, c o m_{k j}^{\prime}\right)$ are equal. Since one can only commit to bits when using $B C^{Q R}$, the equality proof implies that the values $r_{k j}$ in Step 2 were binary. An efficient proof can again be carried out by fairly standard techniques. For instance, one can see two pairs of commitments $B C^{D L}$ and $B C^{Q R}$, where both commitments in one pair contain " 0 " and in the other pair " 1 ", as a cryptographic capsule and proceed similar to [Ben87]. This is a proof with one-bit challenges and thus the least efficient part of our protocol. However, even if one only compares it with the simplest embedding procedure that might follow, fingerprinting without collusion tolerance as in [PS96], one sees that quadratic residue commitments must be made on a portion of the data item significantly larger than the word emb to be embedded into it (so that the resulting changes are small). Thus the complexity of our last step is not larger than that of embedding.

\subsection{Identification}

After finding a redistributed copy of the data item, $\mathcal{M}$ tries to identify a traitor as follows:

Step 1: $\mathcal{M}$ extracts a value $e m b=\left(r_{1}, r_{2}\right)$ from the redistributed data item using the extraction algorithm from the underlying embedding scheme. This pair is $(i s, s)$ with $s \neq 0 . \mathcal{M}$ computes $m^{\prime} \equiv g_{1}^{i s} g_{2}^{s} \bmod p$ and retrieves coin', text, and sig $_{\text {text }}$ from the purchase record of the corresponding data item. If he does not find the coin identifier $m^{\prime}$, he gives up (the collusion tolerance of the underlying code may be exceeded). Otherwise he sends $i$ to $\mathcal{R C}$.

Step 2: $\mathcal{R C}$ searches in its registration database for a buyer who is registered under the value $h_{1} \equiv g_{1}^{i}$. It retrieves the values $\left(I D_{\mathcal{B}}, s g_{\text {coin }}\right)$ and sends them to $\mathcal{M}$. Note that $\mathcal{M}$ can enforce $\mathcal{R C}$ 's cooperation, see below.

Step 3: $\mathcal{M}$ verifies the signature $\operatorname{sig}_{\text {coin }}$ on $h_{1}$.

Enforced Identification This is a special case in identification if $\mathcal{R C}$ refuses to reveal the information requested by $\mathcal{M}$ :

Step 1: $\mathcal{M}$ sends $\operatorname{proof}_{1}=\left(\operatorname{coin}^{\prime},(i, s)\right)$ to an arbiter $\mathcal{A}$.

Step 2: $\mathcal{A}$ verifies the validity of coin' using the algorithm from Step 1 of fingerprinting and that $m^{\prime} \equiv g_{1}^{i s} g_{2}^{s} \bmod p$. If this is wrong, $\mathcal{A}$ rejects $\mathcal{M}$ 's claim. Otherwise she sends $i$ to $\mathcal{R C}$ and requests the values $\left(I D_{\mathcal{B}}, \operatorname{sig}_{\text {coin }}\right)$. Then $\mathcal{A}$ verifies them as $\mathcal{M}$ does in Step 3 of the identification.

\subsection{Trial}

Now $\mathcal{M}$ tries to convince an arbiter $\mathcal{A}$ that $\mathcal{B}$ redistributed the data item bought under the conditions described in text. The values $I D_{\mathcal{B}}$ and text are common inputs. 
Step 1: $\mathcal{M}$ sends to $\mathcal{A}$ the proof string

$$
\text { proof }=\left(\left(i, \operatorname{sig}_{\text {coin }}\right),\left(\operatorname{coin}^{\prime}, \operatorname{sig}_{\text {text }}\right), s\right) \text {. }
$$

Step 2: $\mathcal{A}$ computes $h_{1} \equiv g_{1}^{i} \bmod p$ and verifies that $\operatorname{sig} g_{\text {coin }}$ is a valid signature on $h_{1}$ with respect to $I D_{\mathcal{B}}$. If yes, it means that $i$, the internal structure of an account number $h_{1}$ for which $\mathcal{B}$ was responsible, has been recovered by $\mathcal{M}$ and thus, as we will see, that $\mathcal{B}$ has redistributed some data item. Note that $i$ alone is not enough evidence for $\mathcal{A}$ to find $\mathcal{B}$ guilty of redistributing a data item under the specific text, text.

Step 3: $\mathcal{A}$ verifies the validity of $\operatorname{coin}^{\prime}$, that $m^{\prime} \equiv g_{1}^{i s} g_{2}^{s} \bmod p$ holds, and the signature $s i g_{\text {text }}$ on the disputed text using the test key $p k_{\text {text }}$ contained in coin'. These verifications imply that if the accused buyer owned this coin, she must have spent it in the disputed purchase on text. Now $\mathcal{A}$ must verify that this coin belongs to $\mathcal{B}$. It is not possible to do so by only showing the link between the coin and the withdrawal (which could be fixed by a signature from $\mathcal{B}$ under $\mathcal{R C}$ 's view), because a collusion of $\mathcal{M}$ and $\mathcal{R C}$ could forge such a link. (Interested readers can find the attack in Appendix $\mathrm{A}$ ) Thus $\mathcal{A}$ performs the following last step where $\mathcal{B}$ is required to take part.

Step 4: $\mathcal{A}$ asks $\mathcal{B}$ whether she has withdrawn another valid coin, i.e., a tuple $\operatorname{coin}^{*}=\left(m^{*}, p k_{\text {text }}^{*}, \sigma^{*}\right)$ with $p k_{\text {text }}^{*} \neq p k_{\text {text }}$ using the one-time account $h_{1}$. If yes, $\mathcal{B}$ has to show the representation of $m^{*}$, i.e., a value $s^{*}$ such that $m^{*} \equiv$ $g_{1}^{i s^{*}} g_{2}^{s^{*}}$. If $\mathcal{B}$ can do that, then $\mathcal{A}$ decides that $\mathcal{R C}$ is guilty, otherwise $\mathcal{B}$.

\section{Security of the Construction}

We now present detailed proof sketches of our construction. We assume that all the underlying primitives are secure. The merchant's security only relies on the security of the underlying embedding scheme, the buyer's on standard cryptographic assumptions. The security for the registration center needs the restrictiveness of the blind signature scheme, and we will make a precise assumption for this. Anonymity is information-theoretic.

\subsection{Security for the Merchant}

Due to the properties of the underlying embedding scheme, we can assume that whenever the maximum tolerated size of a collusion is not exceeded, and the collusion redistributes a data item sufficiently similar to the original, then $\mathcal{M}$ can extract a value $e m b$ that belongs to a traitor with very high probability. More precisely $e m b$ is the value to which the traitor could open the final quadraticresidue commitments given in the corresponding purchase.

The zero-knowledge proofs in fingerprinting guarantee that this value $e m b$ is a pair $\left(r_{1}, r_{2}\right)$ such that $g_{1}^{r_{1}} g_{2}^{r_{2}} \equiv m^{\prime} \neq 1$, where $m^{\prime}$ is the identifier of the coin used in this purchase. Thus $\mathcal{M}$ can retrieve a valid coin' and the pair $(i, s) \equiv\left(r_{1} / r_{2}, r_{2}\right)$. This enables him to ask $\mathcal{R C}$ for identification and, in the 
worst case, have it enforced by $\mathcal{A}$. Moreover, he can retrieve text and a valid signature $\operatorname{sig}_{\text {text }}$. Together with the values that $\mathcal{R C}$ must return, $\mathcal{M}$ therefore obtains a valid proof string proof and passes the first three steps of the trial. This is sufficient because the Step 4 of the trial only concerns $\mathcal{A}$ 's decision on whether $\mathcal{R C}$ or $\mathcal{B}$ is guilty.

$\mathcal{M}$ is also protected from making wrong accusations (and thus possibly damaging his reputation): Even if there are more than the tolerated number of traitors, $\mathcal{M}$ 's verifications in identification guarantee that whenever he makes an accusation he will not lose in the trial.

\subsection{Security for the Buyer}

Consider an honest buyer $\mathcal{B}$ and a trial about the purchase on a specific text, text, for which $\mathcal{B}$ has not revealed the corresponding data item. She is secure if the attackers cannot convince an honest arbiter $\mathcal{A}$ in this trial, even if the other parties collude and obtain other data items that she bought (active attack). Such situations occur, e.g., if $\mathcal{B}$ is allowed to redistribute another item after a certain period of time.

Step 2 of the trial guarantees that $\mathcal{B}$ is only held responsible for one of her own one-time account numbers $h_{1} \equiv g_{1}^{i}$, and that the attackers must know $i$.

First it is shown that the attackers cannot find $i$ unless they obtain the result of a purchase where $\mathcal{B}$ has used a coin coin ${ }^{*}$ withdrawn from the account $h_{1}$. The only knowledge the attackers can otherwise obtain about $i$ in our protocol is: (1) $h_{1}$ itself, (2) the proof of knowledge of $i$ in registration, and (3) the commitments on $e m b=(i s, s)$ and two zero-knowledge proofs in fingerprinting. Additionally, they might obtain information in embedding, but by definition of secure embedding this is not the case. If the proofs are actually zero-knowledge and the commitments semantically secure, computing $i$ from all this information is as hard as computing it from $h_{1}$ alone, i.e., as computing a discrete logarithm. (If we use Schnorr identification and a similar proof in fingerprinting, security relies on the joint security of these identification protocols against retrieval of the secret key.)

Hence the only way for the attackers to find $i$ is in fact to obtain the resulting data item in a purchase where $\mathcal{B}$ has used coin ${ }^{*}$ based on $h_{1}$. Let text ${ }^{*}$ be the text describing that purchase. By the precondition, we know that text $\neq$ text. The secret key $s k_{\text {text }}^{*}$ corresponding to $p k_{\text {text }}^{*}$ in coin $^{*}$ is known only to $\mathcal{B}$, and $\mathcal{B}$ reveals no information about it during registration, fingerprinting, and redistribution except making one signature on text* with it. As we assume that the underlying signature scheme is secure against active attacks, this does not help the attackers to forge a valid signature with respect to $p k_{\text {text }}^{*}$ on text.

Now, even if the attackers are a collusion of $\mathcal{M}$ and $\mathcal{R C}$ and succeed in constructing a wrong coin $\operatorname{coin}_{A}$ with a self-made $p k_{\text {text }}^{A}$ which passes all $\mathcal{A}$ 's verifications so far, $\mathcal{B}$ wins by showing $\operatorname{coin}^{*}$ with $p k_{\text {text }}^{*} \neq p k_{\text {text }}^{A}$. 


\subsection{Security for the Registration Center}

An honest registration center $\mathcal{R C}$ should never be found guilty by an honest arbiter $\mathcal{A}$. This could happen in two cases:

1. In enforced identification if $\mathcal{M}$ can send a value proof $f_{1}$ that convinces $\mathcal{A}$, but $\mathcal{R C}$ cannot reveal the required registration data under the identity $i$.

2. In a trial if the attackers (a collusion of $\mathcal{M}$ and $\mathcal{B}$ ) can generate two valid coins, coin' and coin ${ }^{*}$, withdrawn from the same account $h_{1}$, i.e., their representations correspond to the same $h_{1}$.

To exclude both cases, we need the restrictiveness of the underlying blind signature scheme. The corresponding assumption in Bra94 is fairly informal, so we formalize it in a version that suffices for our purpose. Note that although this version seems closest to Brands' formulation, for his payment system a weaker assumption would be sufficient, and the corresponding definition in [FY93] is also of that weaker form: For the payment system one only needs that all the coins the attackers can construct correspond to some account of the attackers. For Case 2 of $\mathcal{R C}$ 's security, we need that the attackers cannot even transform coins between their own accounts. (In Bra94, Definition 5, this is somehow implicit because a one-to-one correspondence between constructed coins and withdrawal protocols is assumed a priori.) We only need the case with one-time accounts, but we formulate the general case, and also for any number of generators. We first introduce some notation:

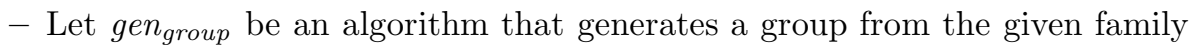
(see Section 4) and a fixed number $n+1$ (in our concrete case $n=2$ ) of random generators $g$ and $g_{1}, \ldots, g_{n}$. Its output $\left(p, q, g, g_{1}, \ldots, g_{n}\right)$ is denoted by desc.

- Let gen $_{k e y}$ be the key generation algorithm that takes as input a value desc and outputs a key pair $(s k, p k)$, here $\left(x, g^{x}\right)$.

- Let valid (desc, $\left.p k,\left(m^{\prime}, p a r, \sigma^{\prime}\right)\right)$ be the predicate for validity of a coin as in Step 1 of fingerprinting (where par may be an arbitrary value in the place of $\left.p k_{t e x t}\right)$.

- By the predicate repr we denote that a vector $I=\left(i_{1}, \ldots, i_{n}\right) \in \mathbb{Z}_{q}^{n}$ is the representation of a message $m \in G_{q}$, i.e.,

$$
\operatorname{repr}(m, I): \Longleftrightarrow m=g_{1}^{i_{1}} \ldots g_{n}^{i_{n}} \text {. }
$$

- blindsig is the protocol between a signer $\mathcal{S}$ and a (dishonest) recipient $\mathcal{R}$ shown in Figure 1 Signer and recipient are interactive probabilistic polynomial-time algorithms, and in our case $\mathcal{S}$ always follows the protocol and $\mathcal{R}$ need not. The signer inputs $s k$ and a message $m$, and the recipient has an arbitrary auxiliary local variable $a u x$ as input; it models the attacker's memory between protocol executions. The signer has no final output, while the recipient outputs an update $a u x^{\prime \prime}$ of its auxiliary variable. If the recipient wants to continue the attack, we also let him immediately output the message 
$m^{\prime \prime}$ that the signer should sign next, and we also allow another output $o^{\prime \prime}$. We write one such interaction as

$$
\left(\left(a u x^{\prime \prime}, m^{\prime \prime}, o^{\prime \prime}\right),-\right) \leftarrow \operatorname{blindsig}(\mathcal{R}(a u x), \mathcal{S}(s k, m)) .
$$

We now consider a dishonest recipient $\mathcal{R}$ who executes the withdrawal protocol with an honest Brands signer $\mathcal{S}$ polynomially many times. In addition, $\mathcal{R}$ has to show a representation $I_{j}$ of each input message $m_{j}$. (Note that we make the assumption with actual outputs in places where the real protocols only have proofs of knowledge.) At the end, $\mathcal{R}$ outputs valid signatures, again with representations of the signed messages. The assumption (or, implicitly, the definition of strong restrictiveness) is that all the output representations are scalar multiples of input representations, and in a kind of one-to-one mapping. To express this easily, let $\langle\ldots\rangle$ be a notation for a multiset (i.e., unordered like a set but possibly with repetition). We use $\subseteq$ to denote the subset relation for multisets, i.e., each element on the left must also occur on the right with at least the same multiplicity. Finally, for any vector (representation) $I \neq 0$ let $\bar{I}$ denote the line it generates, i.e., the set of its scalar multiples.

Assumption 1 (General Strong Restrictiveness) Let $\mathcal{R}$ denote a probabilistic polynomial-time interactive algorithm, $Q$ a polynomial and $l$ a security parameter. Then for all $Q$ and for all $\mathcal{R}$ that interact with the Brands signer for $k=Q(l)$ times:

$$
\begin{aligned}
& P\left(\neg\left(<\overline{I_{1}^{\prime}}, \ldots, \overline{I_{k^{\prime}}^{\prime}}>\subseteq<\overline{I_{1}}, \ldots, \overline{I_{k}}>\right)\right. \\
& \wedge \forall j=1, \ldots, k: \operatorname{repr}\left(m_{j}, I_{j}\right) \\
& \wedge \forall j=1, \ldots, k^{\prime}:\left(\operatorname{repr}\left(m_{j}^{\prime}, I_{j}^{\prime}\right) \wedge \operatorname{valid}\left(\operatorname{desc}, p k,\left(m_{j}^{\prime}, \operatorname{par}_{j}, \sigma_{j}^{\prime}\right)\right)\right) \\
& \wedge \text { all pairs }\left(m_{j}^{\prime}, \text { par }_{j}\right) \text { are different } \\
& :: \quad \text { desc } \leftarrow \operatorname{gen}_{\text {group }}(l) ; k:=Q(l) ;(s k, p k) \leftarrow \operatorname{gen}_{\text {key }}(\text { desc }) ; \\
& \left(\left(\text { aux }_{1}, m_{1}, I_{1}\right),-\right) \leftarrow \mathcal{R}(l, \text { desc }, p k) \text {; } \\
& \left(\left(\operatorname{aux}_{2}, m_{2}, I_{2}\right),-\right) \leftarrow \operatorname{blindsig}\left(\mathcal{R}\left(\operatorname{aux}_{1}\right), \mathcal{S}\left(s k, m_{1}\right)\right) ; \\
& \left(\left(\operatorname{aux}_{k}, m_{k}, I_{k}\right),-\right) \leftarrow \operatorname{blindsig}\left(\mathcal{R}\left(\operatorname{aux}_{k-1}\right), \mathcal{S}\left(s k, m_{k-1}\right)\right) ; \\
& \left(\operatorname{aux}_{k+1},-\right) \leftarrow \operatorname{blindsig}\left(\mathcal{R}\left(\operatorname{aux}_{k}\right), \mathcal{S}\left(s k, m_{k}\right)\right) \text {; } \\
& \left.\left(k^{\prime},\left(\left(m_{1}^{\prime}, \operatorname{par}_{1}, \sigma_{1}^{\prime}\right), \ldots,\left(m_{k^{\prime}}^{\prime}, \operatorname{par}_{k^{\prime}}, \sigma_{k^{\prime}}^{\prime}\right)\right),\left(I_{1}^{\prime}, \ldots, I_{k^{\prime}}^{\prime}\right)\right) \leftarrow \mathcal{R}\left(a u x_{k+1}\right)\right) \\
& \leq 1 / \operatorname{poly}(l) \text {. }
\end{aligned}
$$

Next we return to the security of fingerprinting and consider a successful attacker $\mathcal{R}^{*}$ against $\mathcal{R C}$ 's security. As $\mathcal{R C}$ never uses its secret key except in the blind signature protocol within registration, $\mathcal{R}^{*}$ can be seen as an attacker against the blind signature protocol with $n=2$. The only difference to the scenario in Assumption 1 up to the choice of $\operatorname{aux}_{k+1}$ is that $\mathcal{R}^{*}$ need not output the values $I_{j}$, but instead gives a proof of knowledge of $i_{j}$ in the specific representation 
$m_{j}=g_{1}^{i_{j}} g_{2}$. We combine $\mathcal{R}^{*}$ with the extractor for these proofs of knowledge to obtain another attacker $\mathcal{R}$ that also outputs $I_{j}$, so that Assumption[1 applies.

We now consider the two cases of how $\mathcal{R C}$ could be found guilty by an honest arbiter with the new attacker $\mathcal{R}$.

In Case 1 , the attackers need a valid coin $\operatorname{coin}^{\prime}=\left(m^{\prime}, p k_{\text {text }}, \sigma^{\prime}\right)$ and a representation $I=(i s, s)$ of $m^{\prime}$ such that no withdrawal with respect to $h_{1}=g_{1}^{i}$ was performed. (If $\mathcal{R C}$ does find such a withdrawal it is clear that the retrieved values pass $\mathcal{A}$ 's tests.) However, by Assumption 1, $I$ is a scalar multiple of one of the original representations, say $I_{j}$. As $I_{j}$ is of the form $\left(i_{j}, 1\right)$, this means that $i=i_{j}$ and thus a withdrawal with $h_{1}=g_{1}^{i}$ was performed.

In Case 2, the attackers must show two valid coins $\operatorname{coin}^{\prime}=\left(m^{\prime}, p k_{\text {text }}, \sigma^{\prime}\right)$ and $\operatorname{coin}^{*}=\left(m^{*}, p k_{\text {text }}^{*}, \sigma^{*}\right)$ with $p k_{\text {text }}^{*} \neq p k_{\text {text }}$ and representations $(i s, s)$ of $m^{\prime}$ and $\left(i s^{*}, s^{*}\right)$ of $m^{*}$ with the same $i$. These output representations lie on the same line $\bar{I}$ with $I=(i, 1)$. By Assumption 1 , this $\bar{I}$ and thus $h_{1}=g_{1}^{i}$ also have to occur twice among the input representations. However, $\mathcal{R C}$ ensures that each account is only used once. This finishes the proof that such a successful attacker cannot exist.

\subsection{Anonymity}

Due to the properties of the underlying cash system the views of $\mathcal{R C}$ and $\mathcal{M}$ concerning the withdrawal of a coin and its verification are unlinkable.

We now consider the additional information in registration and fingerprinting: Obviously, sig ${ }_{\text {coin }}$ in registration is no problem because the only additional information it is based on ( $\mathcal{B}$ 's secret key) is not used in fingerprinting. Similarly, sig $_{\text {text }}$ in fingerprinting is no problem: The only additional information it is based on is $s k_{\text {text }}$. However, an information-theoretic attacker can compute $s k_{\text {text }}$ from $p k_{\text {text }}$, which is already transmitted in the coin, and we know that the coin-related data in the underlying cash system are not linkable.

The value $e m b$ in fingerprinting, which is based on the same $i$ that is also used in fingerprinting, is hidden in bit commitments. The first commitment scheme used, based on discrete logarithms, is information-theoretically hiding and the second one, based on quadratic residues, is hiding under the Quadratic Residuosity Assumption. Moreover the zero-knowledge protocols used in fingerprinting do not leak information about emb and the embedding operation is assumed not to leak such information either.

Furthermore using one-time accounts implies that $\mathcal{B}$ 's different purchases are unlinkable, even if a purchased data item has been redistributed and the information contained in it recovered.

\section{Conclusion}

We have presented an anonymous fingerprinting scheme where all protocols are explicit and fairly efficient. The complexity is lower than that of any known embedding procedure that might follow, so that the anonymity is currently not 
the bottleneck for implementation. A disadvantage of this scheme in comparison with the previous one based on general zero-knowledge techniques is that the buyer is needed to carry out a fair trial; we hope to find a scheme that combines explicitness and 2-party trials in the future.

Of course, embedding for any asymmetric scheme is still rather an expensive procedure because commitments on a significant fraction of the data are needed, and the data must be long because otherwise one could not hide enough bits in them, at least if one desires collusion tolerance.

Acknowledgments: We thank Matthias Schunter and Michael Waidner for generous and fruitful discussions.

\section{References}

[BCC88] Gilles Brassard, David Chaum, Claude Crépeau: Minimum Disclosure Proofs of Knowledge; Journal of Computer and System Sciences 37 (1988) 156-189.

[BCP88] Jurjen Bos, David Chaum, George Purdy: A Voting Scheme; unpublished manuscript, presented at the rump session of Crypto' 88 .

[Ben87] Josh Cohen Benaloh: Cryptographic Capsules: A Disjunctive Primitive for Interactive Protocols; Crypto'86, LNCS 263, Springer-Verlag, Berlin 1987, 213-222.

[BKK90] Joan F. Boyar, Stuart A. Kurtz, Mark W. Krentel: A Discrete Logarithm Implementation of Perfect Zero-Knowledge Blobs; Journal of Cryptology $2 / 2$ (1990) 63-76.

[BM97] Ingrid Biehl, Bernd Meyer: Protocols for Collusion-Secure Asymmetric Fingerprinting; STACS 97, LNCS 1200, Springer-Verlag, Berlin 1997, 399-412.

[BMP86] G. R. Blakley, Catherine Meadows, George B. Purdy: Fingerprinting Long Forgiving Messages; Crypto'85, LNCS 218, Springer-Verlag, Berlin 1986, 180-189.

[Bra94] Stefan Brands: Untraceable Off-line Cash in Wallet with Observers; Crypto'93, LNCS 773, Springer-Verlag, Berlin 1994, 302-318.

[BS95] Dan Boneh, James Shaw: Collusion-Secure Fingerprinting for Digital Data; Crypto'95, LNCS 963, Springer-Verlag, Berlin 1995, 452-465.

[CEG88] David Chaum, Jan-Hendrik Evertse, Jeroen van de Graaf: An Improved Protocol for Demonstrating Possession of Discrete Logarithms and some Generalizations; Eurocrypt'87, LNCS 304, Springer-Verlag, Berlin 1988, 127-141.

[CFN94] Benny Chor, Amos Fiat, Moni Naor: Tracing Traitors; Crypto'94, LNCS 839, Springer-Verlag, Berlin 1994, 257-270.

[CHP92] David Chaum, Eugène van Heijst, Birgit Pfitzmann: Cryptographically Strong Undeniable Signatures, Unconditionally Secure for the Signer; Crypto'91, LNCS 576, Springer-Verlag, Berlin 1992, 470-484.

[CP93] David Chaum, Torben Pryds Pedersen: Wallet Databases with Observers; Crypto'92, LNCS 740, Springer-Verlag, Berlin 1993, 89-105.

[Dom98] Josep Domingo-Ferrer: Anonymous Fingerprinting of Electronic Information with Automatic Identification of Redistributors; Electronics Letters 34/13 (1998) 1303-1304.

[FN94] Amos Fiat, Moni Naor: Broadcast Encryption; Crypto'93, LNCS 773, Springer-Verlag, Berlin 1994, 480-491. 
[FTY96] Yair Frankel, Yiannis Tsiounis, Moti Yung: "Indirect Discourse Proofs": Achieving Efficient Fair Off-Line E-cash; Asiacrypt'96, LNCS 1163, Springer-Verlag, Berlin 1997, 287-300.

[FY93] Matthew Franklin, Moti Yung: Secure and Efficient Off-Line Digital Money; 20th International Colloquium on Automata, Languages and Programming (ICALP), LNCS 700, Springer-Verlag, Berlin 1993, 265-276.

[GM84] Shafi Goldwasser, Silvio Micali: Probabilistic Encryption; Journal of Computer and System Sciences 28 (1984) 270-299.

[KD98] Kaoru Kurosawa, Yvo Desmedt: Optimum Traitor Tracing and Asymmetric Schemes; Eurocrypt'98, LNCS 1403, Springer-Verlag, Berlin 1998, 145157.

[NP98] Moni Naor, Benny Pinkas: Threshold Traitor Tracing; Crypto'98, LNCS 1462, Springer-Verlag, Berlin 1998, 502-517.

[Ped92] Torben Pryds Pedersen: Non-Interactive and Information-Theoretic Secure Verifiable Secret Sharing; Crypto'91, LNCS 576, Springer-Verlag, Berlin 1992, 129-140.

[Pfi96] Birgit Pfitzmann: Trials of Traced Traitors; Information Hiding, LNCS 1174, Springer-Verlag, Berlin 1996, 49-64.

[PS96] Birgit Pfitzmann, Matthias Schunter: Asymmetric Fingerprinting; Eurocrypt'96, LNCS 1070, Springer-Verlag, Berlin 1996, 84-95.

[PW97a] Birgit Pfitzmann, Michael Waidner: Asymmetric Fingerprinting for Larger Collusions; 4th ACM Conference on Computer and Communications Security, acm press, New York 1997, 151-160.

[PW97b] Birgit Pfitzmann, Michael Waidner: Anonymous Fingerprinting; Eurocrypt'97, LNCS 1233, Springer-Verlag, Berlin 1997, 88-102.

[Sch91] Claus-Peter Schnorr: Efficient Signature Generation by Smart Cards; Journal of Cryptology 4/3 (1991) 161-174.

\section{A Linking a Self-Made Coin to a Correct Withdrawal}

In this appendix, we show the attack that motivates the last verification step in the trial, as mentioned in Section 4.4.

Let $h_{1}$ be an account number of $\mathcal{B}$ and $\operatorname{coin}^{*}=\left(m^{\prime}, p k_{\text {text }}^{*}, \sigma^{\prime}\right)$ (with $\sigma^{\prime}=$ $\left.\left(z^{\prime}, a^{\prime}, b^{\prime}, r^{\prime}\right)\right)$ be the coin that $\mathcal{B}$ withdrew from this account and used in a purchase with the text text*. Note that $a^{\prime} \equiv a^{u} g^{v}=g^{u w+v} \bmod p$ and $b^{\prime} \equiv$ $b^{s u} m^{\prime v}=m^{w s u} m^{\prime v}=m^{\prime w u+v} \bmod p$.

Assume now that the attackers are a collusion of $\mathcal{M}$ and $\mathcal{R C}$ and have access to the data item that $\mathcal{B}$ has bought in this purchase. Then they know emb= $(i s, s), w, x$, view $_{\text {withdraw }}=(a, b, c), r$, and the additional signature sig $_{\text {coin }}^{*}$ that $\mathcal{B}$ would give to fix view withdraw before receiving $r$.

As $\operatorname{sig}_{\text {coin }}^{*}$ fixes the values $(a, b, c)$ that can be used together with the given $h_{1}$, the attackers want to link a self-made coin $\operatorname{coin}_{A}^{\prime}$, in particular with a selfmade $p k_{\text {text }}^{A}$ for which the attackers know $s k_{\text {text }}^{A}$, to these values. For this, they randomly select $w_{A} \in_{R} \mathbb{Z}_{q}$ and compute $a_{A}^{\prime} \equiv g^{w_{A}} \bmod p$ and $b_{A}^{\prime} \equiv m^{\prime w_{A}} \bmod p$. Then they compute $c_{A}^{\prime}=\operatorname{hash}\left(m^{\prime}, z^{\prime}, a_{A}^{\prime}, b_{A}^{\prime}, p k_{\text {text }}^{A}\right)$. Since $c$ is fixed they first compute $u_{A} \equiv c_{A}^{\prime} / c \bmod q$ and then $v_{A} \equiv w_{A}-w u_{A}$ and $r_{A}^{\prime} \equiv r u_{A}+v_{A} \bmod q$. In this manner they obtain values which pass all verifications by $\mathcal{A}$.

Finally, the attackers can use $s k_{\text {text }}^{A}$ to sign any text they like with respect to this coin. 